\title{
Reprise of the Supershells
}

\author{
Sayan Chakraborti ${ }^{1}$ and Alak Ray ${ }^{2}$ \\ ${ }^{1}$ Harvard University, Cambridge MA 02138, USA \\ email: schakraborti@fas.harvard.edu \\ ${ }^{2}$ Tata Institute of Fundamental Research, Mumbai 400005, India \\ email: akr@tifr.res.in
}

\begin{abstract}
Neutral hydrogen cavities have been detected in the Milky Way for decades and more recently in other nearby star-forming galaxies. It has been suggested that at least a fraction of them may be expanding supershells driven by the combined mechanical feedback from multiple supernovae occurring in an OB association. Yet most extragalactic cavities had neither a demonstrated expansion velocity nor an identified OB association inside them. In this presentation, we will outline how new multiwavelength observations are providing us with systems to test the theory of supershells driven by the mechanical feedback from multiple supernovae. We shall also discuss the consequences of these recent results in the context of supernova feedback, propagating star formation and particle acceleration.
\end{abstract}

Keywords. supernovae: general - Galaxies: individual (M101) - ISM: Bubbles — Radio lines: ISM

\section{Intorduction}

Most massive stars in spiral galaxies are born in OB associations and other young stellar clusters. They contain a few to thousands of massive stars of spectral class $\mathrm{O}$ and B. O stars are short-lived, and die as supernovae within a few megayears. These associations last for few tens of megayears because all their massive stars exhaust their nuclear fuel within this duration. OB associations ionize the interstellar medium (ISM) in galaxies and produce localized HII regions as well as diffuse ionized gas. According to Oey et al. (2002) supernovae, exploding from the massive stars that form in these associations, pressurize and churn the ISM. Expanding neutral hydrogen structures, called supershells were identified in the Milky Way by Heiles (1979). McCray and Kafatosw (1987) have shown that supershells form and evolve due to the continuous mechanical energy injection by stellar winds and $\mathrm{SNe}$ in massive parent $\mathrm{OB}$ associations. The outer shocks of the superbubbles sweep up the ambient ISM into a thin cool shell. These supershells may also play a role in the process of star formation and favor propagating star formation, as pointed out by McCray and Kafatosw (1987), Palous et al. (1994). Hopkins et al. (2008), point out that the neutral and molecular gas replenishment in the walls of the supershells may provide the trigger for collapse and further star formation. In NGC 300, Blair and Long (1997) reported several cavities, much larger than galactic supernova remnants (SNRs). Payne et al. (2004) suggest that these may represent the above mentioned supershells.

Cavities have been found in neutral hydrogen (HI) surface density maps of several nearby galaxies. For a classical review and inventory of HI holes, see Tenorio-Tagle and Bodenheimer (1988). For a recent review and survey of HI holes see Bagetakos et al. (2011). Also seeKamphuis et al. (1991), Chakraborti and Ray (2011) for supershells in 

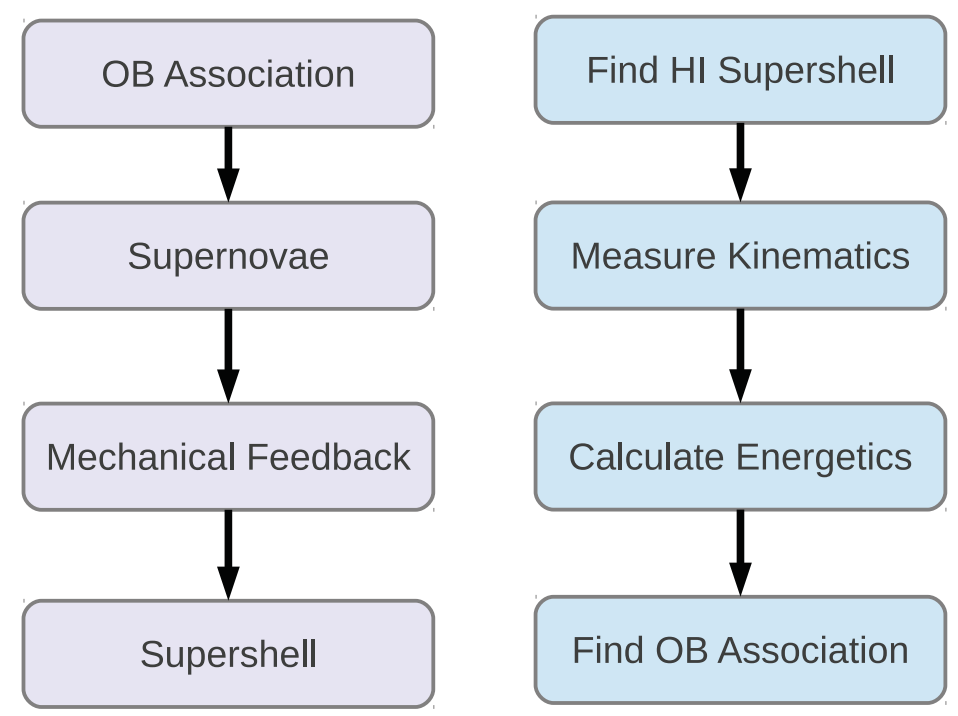

Figure 1. The flowchart on the left describes how a supershell is formed. It starts with an initial OB association which finally powers the supershells. The flowchart on the right describes how one can test this paradigm. It starts by finding a superhsell and looks for its energy source, namely an $\mathrm{OB}$ association. Note that the steps in the two charts essentially go in reverse.

M101. However, the presence of a cavity alone is insufficient evidence for the existence of a supershell. It may simply be a low density region between higher density ones, created by turbulent distribution of gas. This pitfall is illustrated by the case of Holmberg II. In that galaxy Puche et al. (1992) used underdensities in HI column density map to create a catalog of $\mathrm{HI}$ cavities and postulated that they have been evacuated by multiple SNe. However Rhode et al. (1999) rule out a multiple supernova origin for most of these holes since they were unable to find any young cluster or even trace of recent star formation activity which would have led to the SNe responsible for these HI-holes. Stewart et al. (2000) have since then re-examined the Rhode et al. (1999) results and concluded that the energy available from massive stars inside HI shells indicates that energy deposited into the ISM from supernovae and stellar winds is sufficient to account for the HI morphology. Silich et al. (2006) have found that the observed properties of the most prominent kpc-scale structure in IC 1613 and the level of the detected star formation activity are inconsistent with the hypothesis that they are formed by energy injection from multiple supernovae.

The first unambiguous case of an extragalactic expanding HI shell associated with a hole in the HI surface density was reported by Kamphuis et al. (1991) in observations of M101 using the Westerbork Synthesis Radio telescope. Weisz et al. (2009) have investigated the creation of a supergiant HI shell in a dwarf irregular galaxy IC 2574 and its role in triggering star formation around it. Star formation studies such as those reviewed by Kennicutt (1998) in most nearby galaxies indicate that sites of recent star formation are correlated with sites of higher HI surface densities, leading to several recipes for star formation. The feedback processes described above should however clear out the gas and lead to young stellar associations in regions of low gas density surrounded by higher density swept up shells. Therefore we began searching for cavities in the HI surface density, occupied by young clusters. 


\section{Our Work}

Chakraborti and Ray (2011) report yet another striking case of an expanding HI supershell in M101 which we argue is driven by multiple supernovae from a young stellar association. We use the observed properties of the HI supershell to determine its dynamical age and energy requirement. Here we demonstrate that this HI cavity has measured expansion velocity and at the same time is fully contained, i.e. it has not yet broken out of the disk of its host galaxy. We also detect a UV emitting stellar association inside the HI hole. We model its spectral energy distribution, test and confirm the hypothesis of McCray and Kafatosw (1987) that some supershells are driven by the combined action of multiple supernovae occurring in a stellar association inside it.

Our approach has been to first find the supershells from $21 \mathrm{~cm}$ (neutral Hydrogen) observations of the host galaxy. We define a HI supershells as an underdensity of neutral hydrogen where we can detect both an approching and receeding component. We use data from The HI Nearby Galaxy Survey (THINGS) undertaken by Walter et al. (2008) at the NRAO Very Large Array (VLA) to study the HI emission from nearby galaxies. The high angular resolution $\left(\sim 7^{\prime \prime}\right)$ and high spectral resolution $(\sim 5 \mathrm{~km} / \mathrm{s})$ data allow us to measured the size $\left(R_{S}\right)$ and expansion velocity $\left(V_{S}\right)$ of the supershell.

We then inverted the McCray and Kafatosw (1987) model, as $R_{S}$ and the $V_{S}$ are directly observable from the HI data. In this work, we therefore re-frame the equations as

$$
t_{7}=\left(R_{S} / 97 \mathrm{pc}\right)\left(V_{S} / 5.7 \mathrm{~km} \mathrm{~s}^{-1}\right)^{-1}
$$

and

$$
\left(N_{*} E_{51} / n_{0}\right)=\left(R_{S} / 97 \mathrm{pc}\right)^{2}\left(V_{S} / 5.7 \mathrm{~km} \mathrm{~s}^{-1}\right)^{3}
$$

to express the variables, which characterize the supershell, purely in terms of the observable quantities. These equations will allow us to estimate the age $\left(t=t_{7} \times 10^{7}\right.$ years $)$ and mass (where $N_{*}$ is the number of massive stars) of an association which will suffice to reproduce the observed size and expansion velocity of any given supershell. We used data from Far and Near Ultraviolet observations are available from GALEX Nearby Galaxies Survey, conducted by Martin et al. (2005) with the Galaxy Evolution Explorer (GALEX). The ultraviolet emission provided us with a simple tool for identifying the young stellar association, which was supplied the energy budget for the expanding supershell.

\section{The way ahead}

It is important to understand supernova feedback in galaxies at from the level of individual supernovae to the whole galaxy. Supershells a a very important link in this chain and Chakraborti and Ray (2011) have shown that the dynamics of a supernova driven supershell can explain the kinematics of an observed supershell. Chakraborti (2011) shows that self-regulated star formation, driven by the competition between gravitational instabilities and mechanical feedback from supernovae, can explain the nearly constant neutral hydrogen surface density across galaxies. Warren et al. (2011) studied the formation of kiloparsec-scale HI holes in dwarf galaxies and concluded that large HI holes are likely formed from multiple generations of star formation. This complicates the story of a single coeval star cluster powering a single supershell. SN 2008jb, discovered by Prieto et al. (2012), was the first supernova inside a supershell. This is our opportunity to see supernova feedback live in action. Suad et al. (2012) reported the discovery of GS10002-41, yet another large HI shell in the outer Milky Way and discussed the evidence for trigerred star formation. This goes on to show that new supershells can still be found 
in our own galaxy and as a result studied in great detail. Cold filamentary structures formed in supershells, simulated by Ntormousi et al. (2011), could be responsible for trigerred star formation. The study of Continuum Halos in Nearby Galaxies: An EVLA Survey (CHANG-ES) led by Irwin et al. (2012) may open new windows into magnetized outflows from large supershells bursting out of the cold gas disk of starforming galaxies. Roy et al. (2013) outline the conditions necessary for such break outs.

\section{References}

M. S. Oey, B. Groves, L. Staveley-Smith, \& R. C. Smith, AJ 123, 255-268 (2002), arXiv: astro-ph/0112057

C. Heiles, ApJ 229, 533-537 (1979)

R. McCray, \& M. Kafatos, ApJ 317, 190-196 (1987)

J. Palous, G. Tenorio-Tagle, \& J. Franco, MNRAS 270, 75 (1994)

A. M. Hopkins, N. M. McClure-Griffiths, \& B. M. Gaensler, ApJ 682, L13-L16 (2008), 0806.0662

W. P. Blair, \& K. S. Long, ApJS 108, 261 (1997)

J. L. Payne, M. D. Filipović, T. G. Pannuti, P. A. Jones, N. Duric, G. L. White, \& S. Carpano, A\& A 425, 443-456 (2004)

G. Tenorio-Tagle, \& P. Bodenheimer, ARA\& A 26, 145-197 (1988)

I. Bagetakos, E. Brinks, F. Walter, W. J. G. de Blok, A. Usero, A. K. Leroy, J. W. Rich, \& R. C. Kennicutt, Jr., $A J$ 141, 23 (2011), 1008.1845

J. Kamphuis, R. Sancisi, \& T. van der Hulst, A\& A 244, L29-L32 (1991)

S. Chakraborti, \& A. Ray, ApJ 728, 24 (2011), 1011.5232

D. Puche, D. Westpfahl, E. Brinks, \& J.-R. Roy, AJ 103, 1841-1858 (1992)

K. L. Rhode, J. J. Salzer, D. J. Westpfahl, \& L. A. Radice, AJ 118, 323-336 (1999), arXiv:astro-ph/9904065

S. G. Stewart, M. N. Fanelli, G. G. Byrd, J. K. Hill, D. J. Westpfahl, K.-P. Cheng, R. W. O'Connell, M. S. Roberts, S. G. Neff, A. M. Smith, \& T. P. Stecher, ApJ 529, 201-218 (2000)

S. Silich, T. Lozinskaya, A. Moiseev, N. Podorvanuk, M. Rosado, J. Borissova, \& M. ValdezGutierrez, A\& A 448, 123-131 (2006), arXiv:astro-ph/0510812

D. R. Weisz, E. D. Skillman, J. M. Cannon, F. Walter, E. Brinks, J. Ott, \& A. E. Dolphin, ApJ 691, L59-L62 (2009), 0812.2036

R. C. Kennicutt, Jr., ARA\& A 36, 189-232 (1998), arXiv:astro-ph/9807187

F. Walter, E. Brinks, W. J. G. de Blok, F. Bigiel, R. C. Kennicutt, Jr., M. D. Thornley, \& A. Leroy, $A J 136,2563-2647$ (2008), 0810.2125

D. C. Martin, J. Fanson, D. Schiminovich, P. Morrissey, P. G. Friedman, T. A. Barlow, T. Conrow, R. Grange, P. N. Jelinsky, B. Milliard, O. H. W. Siegmund, L. Bianchi, Y.-I. Byun, J. Donas, K. Forster, T. M. Heckman, Y.-W. Lee, B. F. Madore, R. F. Malina, S. G. Neff, R. M. Rich, T. Small, F. Surber, A. S. Szalay, B. Welsh, \& T. K. Wyder, ApJ 619, L1-L6 (2005), arXiv: astro-ph/0411302

S. Chakraborti, ApJ 732, 105 (2011), 1103.2763

S. R. Warren, D. R. Weisz, E. D. Skillman, J. M. Cannon, J. J. Dalcanton, A. E. Dolphin, R. C. Kennicutt, Jr., B. Koribalski, J. Ott, A. M. Stilp, S. D. Van Dyk, F. Walter, \& A. A. West, ApJ 738, 10 (2011), 1105.4117

J. L. Prieto, J. C. Lee, A. J. Drake, R. McNaught, G. Garradd, J. F. Beacom, E. Beshore, M. Catelan, S. G. Djorgovski, G. Pojmanski, K. Z. Stanek, \& D. M. Szczygieł, ApJ 745, 70 (2012), 1107.5043

L. A. Suad, S. Cichowolski, E. M. Arnal, \& J. C. Testori, AE A 538, A60 (2012), 1112.0516

E. Ntormousi, A. Burkert, K. Fierlinger, \& F. Heitsch, ApJ 731, 13 (2011), 1011.5751

J. Irwin, R. Beck, R. A. Benjamin, R.-J. Dettmar, J. English, G. Heald, R. N. Henriksen, M. Johnson, M. Krause, J.-T. Li, A. Miskolczi, S. C. Mora, E. J. Murphy, T. Oosterloo, T. A. Porter, R. J. Rand, D. J. Saikia, P. Schmidt, A. W. Strong, R. Walterbos, Q. D. Wang, \& T. Wiegert, AJ 144, 43 (2012), 1205.5694

A. Roy, B. B. Nath, P. Sharma, \& Y. Shchekinov, ArXiv e-prints (2013), 1303.2664 


\section{Discussion}

Koo: The $(\mathrm{P}, \mathrm{V})$ diagram that you showed clearly shows that there is a bubble at the position of the supershell. But it is not obvious to me if it shows that these are expanding portions of the shell because these are comparable HI emission at the same velocity outside the supershell.

Chakraborti: Yes, so the point is that this supershell is not so big that it has yet punctured out of the disk of the galaxy. There is an example of a supershell in M101, by Kamphuis et al. (1991 A \& A), in which these components have gone out and detached themselves from the rest of the galaxy. So, that would be an example of a supershell which is so big that it has punctured out of the disk of the galaxy and has fragmented the top and bottom parts bue to Rayleigh Taylor instabilities, possibly, which was discussed by Dr. Biman Nath (see paper by B. Nath at same symposium), but this shell is not yet so big.

FRANCE: Comment: UV spectroscopic observations of hot star wind lines (e.g. CIII $\lambda$ 1177,NV 1240, SiIV 1400, CIV $1550 \AA$ A) will give you a good measure of both age and metallicity when compared with star bursts 99 models.

Chakraborti: There is not much extinction which would stop the UV. So sure, that would be very nice.

WANG: M101 has been well observed in X-ray. This should give you a direct check of your scenario.

Chakraborti: Yes, that is an excellent suggestion. This galaxy has mega-seconds of Chandra observations. We looked at this region of the data. I cant tell you for sure whether there is extended emission there? There seem to be a few point sources. It would be very interesting to be able to detect the extended emission from the coronal gas inside. The point sources would most probably be HMXBs. But this is work which is ongoing and very important to do. 